\section{Antiphosphatidylserine/Prothrombin Antibodies in Systemic Lupus Erythematosus}

\section{To the Editor:}

We read with interest the report by Akhter, et $a l^{1}$ on antiphosphatidylserine/prothrombin (aPS/PT) and IgA antiphospholipid assays in systemic lupus erythematosus (SLE). While we commend the authors on the number of patient samples investigated and fully agree on the importance of aPS/PT as markers of thrombosis in antiphospholipid syndrome (APS), we cannot overlook the weak design of the study.

The authors offer testing for aPS/PT as an "alternative" assay to predict risk of thrombosis in anticoagulated patients where, presumably, the lupus anticoagulant (LAC) testing is not valid. While the invalidity of the LAC testing in anticoagulated patients is a matter of debate ${ }^{2,3,4}$, Akhter, et al supported this hypothesis and found their conclusions in categorical data and OR obtained by univariate analysis, when comparing a population of LAC-positive versus LAC-negative patients. Although all other antibodies tested, such as anticardiolipin antibodies (aCL), anti- $\beta_{2}$-GPI, IgG anti- $\beta_{2}$-GPI DI, and IgA anti- $\beta_{2}$-GPI D4/5, showed an increased OR for LAC, the authors arbitrarily chose to base their conclusions only on the aPS/PT findings, disregarding that anti- $\beta_{2}$-GPI shows even higher significance than aPS/PT, when studying their association with thrombosis.

Moreover, the lack of data on the anticoagulant treatment, unfortunately, weakens even more the authors' strong statements on the potential use of aPS/PT in this setting, because we are left in the dark on the number of patients receiving such treatment.

In 2010, Atsumi and Koike ${ }^{5}$ reported that most of the APS patients with aPS/PT from their cohort had a positive LAC (44/47 in the APS group but $6 / 11$ in the non-APS group), regardless of their anticoagulation status, suggesting that aPS/PT could be a "confirming" assay for APS-associated $\mathrm{LAC}^{6}$.

Recently, we published our data on aPS/PT and its association with $\mathrm{LAC}^{7}$. Our results show that although the association of aPS/PT with thrombosis is stronger in patients with positivity for LAC, the risk of thrombosis is also increased in those without LAC but positive for aPS/PT. After multivariate analysis, our data confirm that the aPS/PT associations with clinical features of APS are independent of the presence of LAC. In addition, we evaluated the clinical accuracy of several aPL specificities, both individually and in combination, in a wide cohort of patients with SLE in an attempt to identify a panel of tests that could provide the best accuracy for diagnosing APS. We showed that a panel including LAC, anti- $\beta_{2}$-GPI, and aPS/PT improved the diagnostic power and helped in stratifying the risk for each patient. Each further aPL positivity detection increased the risk for thrombosis, with OR ranging from 3 to 7 for the single positivity for anti- $\beta_{2}$-GPI and aPS/PT, respectively, to 23 for the triple positivity including the $\mathrm{LAC}^{8}$. Even more, aPS/PT was 1 of the 6 variables along with arterial hypertension, hyperlipidemia, aCL, LAC, and anti- $\beta_{2}$-GPI identified as independent risk factors for APS in the development of a quantitative risk score system, the so-called global APS score ${ }^{9}$.

Overall, we confirm the importance of aPS/PT testing in APS and we hope the authors are able to substantiate their findings after applying multivariate analysis in this very cohort. In addition, we do not propose testing for aPS/PT as an "alternative" but as an additional test for the recognition of the APS.
MARIA LAURA BERTOLACCINI, PhD; SAVINO SCIASCIA, MD, Lupus Research Unit, The Rayne Institute, Division of Women's Health, King's College London; GIOVANNI SANNA, MD, FRCP, PhD, Louise Coote Lupus Unit, Guy's and St. Thomas's NHS Foundation Trust; MUNTHER A.

KHAMASHTA, MD, FRCP, PhD, Lupus Research Unit, The Rayne Institute, Division of Women's Health, King's College London, and Louise Coote Lupus Unit, Guy's and St. Thomas's NHS Foundation Trust, St.

Thomas's Hospital, London, UK. Address correspondence to Dr. M. Bertolaccini, Lupus Research Unit, The Rayne Institute, Division of Women's Health, King's College London, 4th Floor Lambeth Wing, St. Thomas's Hospital, London SE1 7EH, UK. E-mail:

maria.bertolaccini@kcl.ac.uk

Dr. Bertolaccini is funded by the Louise Gergel Fellowship and the St. Thomas's Lupus Trust.

\section{REFERENCES}

1. Akhter E, Shums Z, Norman GL, Binder W, Fang H, Petri M. Utility of antiphosphatidylserine/prothrombin and $\operatorname{IgA}$ antiphospholipid assays in systemic lupus erythematosus. J Rheumatol 2013;40:282-6.

2. Tripodi A, Chantarangkul V, Clerici M, Mannucci PM. Laboratory diagnosis of lupus anticoagulants for patients on oral anticoagulant treatment. Performance of dilute Russell viper venom test and silica clotting time in comparison with Staclot LA. Thromb Haemost 2002;88:583-6.

3. Olteanu H, Downes KA, Patel J, Praprotnik D, Sarode R. Warfarin does not interfere with lupus anticoagulant detection by dilute Russell's viper venom time. Clin Lab 2009;55:138-42.

4. Ortel TL. Antiphospholipid syndrome: laboratory testing and diagnostic strategies. Am J Hematol 2012;87 Suppl 1:S75-81.

5. Atsumi T, Koike T. Antiprothrombin antibody: Why do we need more assays? Lupus 2010;19:436-9.

6. Bertolaccini ML, Amengual O, Atsumi T, Binder WL, de Laat B, Forastiero R, et al. 'Non-criteria' aPL tests: Report of a task force and preconference workshop at the 13th International Congress on Antiphospholipid Antibodies, Galveston, TX, USA, April 2010. Lupus 2011;20:191-205.

7. Bertolaccini ML, Sciascia S, Murru V, Garcia-Fernandez C, Sanna G, Khamashta MA. Prevalence of antibodies to prothrombin in solid phase (aPT) and to phosphatidylserine-prothrombin complex (aPS/PT) in patients with and without lupus anticoagulant. Thromb Haemost 2013;109:207-13.

8. Sciascia S, Murru V, Sanna G, Roccatello D, Khamashta MA, Bertolaccini ML. Clinical accuracy for diagnosis of antiphospholipid syndrome in systemic lupus erythematosus: Evaluation of 23 possible combinations of antiphospholipid antibody specificities. J Thromb Haemost 2012;10:2512-8.

9. Sciascia S, Sanna G, Murru V, Roccatello D, Khamashta MA, Bertolaccini ML. GAPSS: The Global Anti-Phospholipid Syndrome Score. Rheumatology 2013 Jan 12. [E-pub ahead of print]

J Rheumatol 2013;40:9; doi:10.3899/jrheum.130295 